\title{
Paroxysmal Atrial Fibrillation by ECG Finding
}

National Cancer Institute

\section{Source}

National Cancer Institute. Paroxysmal Atrial Fibrillation by ECG Finding. NCI Thesaurus.

Code C120615.

An electrocardiographic tracing demonstrating intermittent atrial fibrillation in the presence of sinus rhythm, junctional rhythm, or an atrial paced rhythm. 